\title{
Search for neutrinos associated with solar flare
}

\author{
Kohei Okamoto, ${ }^{a, 1, *}$ Yuuki Nakano ${ }^{b, 1}$ and and Shintaro Ito ${ }^{c, 1}$ \\ ${ }^{a}$ Kamioka observatory, \\ Higashi-Mozumi 456, Kamioka-cho, Hida-city, Gifu, 506-1205, Japan \\ ${ }^{b}$ Kobe University, \\ Department of Physics, Kobe, Hyogo, 657-8501, Japan \\ ${ }^{c} K E K$, \\ 1-1 Oho, Tsukuba, Ibaraki, 305-0801, Japan \\ E-mail: okamoto@km.icrr.u-tokyo.ac.jp, ynakano@phys.sci.kobe-u.ac.jp, \\ sito@post.kek.jp
}

Importance of search for neutrinos generated during solar flares has been discussed for last 60 years, however, neutrinos associated with solar flares (solar flare neutrinos) have not been obserbed yet. Since neutrinos are not affected by interplanetary magnetic field, solar flare neutrinos would provide us with information about a particle acceleration mechanism in solar flares. According to some theoretical predictions, flux of the solar flare neutrino would depend on the releasing energy and the location where solar flares ocurr on the Sun surface. Typical predicted probability of detection by Super-Kamiokande (SK) detector is $8.5 \times 10^{-1}$ event/flare for a solar flare which occurs on the opposite side of Sun surface from the earth (invisible side). On the other hands, $1.36 \times 10^{-4}$ event/flare would be predicted for the other side (visible side). To minimize background for the solar flare neutrino searches, data of solar satellites (GOES, RHESSI, and Geotail) were analyzed and time windows for solar flare neutrino searches on the visible side were defined. Coronal Mass Ejection event catalogs were used to determine the search windows for solar flare neutrinos on the invisible side of the Sun. SK is the world's largest underground water Cherenkov detector. The SK experiment has been started the measurement of neutrinos since 1996. The results of solar flare neutrino searches using data sets from SK-I to SK-IV are presented.

$37^{\text {th }}$ International Cosmic Ray Conference (ICRC 2021)

July 12 th - 23rd, 2021

Online - Berlin, Germany

${ }^{1}$ For the Super-Kamiokande Collaboration.

*Presenter 


\section{Introduction}

Solar flares convert magnetic energy into plasma heating and the kinetic energy of charged particles such as protons. Protons injected downwards from the coronal acceleration region will interact with the dense plasma in the lower solar atmosphere.

Neutrinos associated with solar flares (solar flare neutrinos) attract significant attention in the field of astrophysics. If protons are accelerated during solar flares to sufficiently high energies (more than $300 \mathrm{MeV}$ ), pions are produced by collisions with other nuclei in the solar atmosphere [1]. Finally, neutrinos are generated via the decay of those charged pions through the same processes as atmospheric neutrinos. Because of this hadronic origin, solar flare neutrinos give information about the acceleration of protons as well as subsequent interactions in the solar chromosphere. For the last 60 years, solar flare neutrinos have been experimentally sought by several neutrino detectors [2] but not observed yet.

There are mainly three predictions for solar flare neutrino flux at the Earth with different assumptions. The flux of solar flare neutrinos depends on the condition of the proton acceleration in solar flares which appear as power index of proton energy distribution. Generation of solar flare neutrinos was simulated assuming the isotropic (visible side) and beam-like (invisible side) proton acceleration with the Monte Carlo methods by Kocharov, Kovaltsov, and Usoskin [3], and Takeishi et al. [4]. For Fargion's and Moscato's prediction [5], the neutrino flux was estimated using the amount of energy taken up by pion production out of the total energy of solar flares. It is assumed that the amount of energy carried away varies depending on whether the accelerated protons are headed for the solar surface or the solar atmosphere. The conditions and the number of expected event observed by the Super-Kamiokande (SK) detector in each prediction are summarized in Table 1.

Table 1: Summary of the predicted solar flare models.

\begin{tabular}{|c||c|c|c|}
\hline Model & $\begin{array}{c}\text { Power index } \\
\text { of parent proton }\end{array}$ & $v$ generation condition & $\begin{array}{c}\text { The number of expected } \\
\text { event in SK [/flare] }\end{array}$ \\
\hline \hline \multirow{2}{*}{ Ref. [3] } & $3-4$ & isotropic & $1.36 \times 10^{-4}$ \\
\cline { 2 - 4 } & 1 & beam like & 0.85 \\
\hline \multirow{2}{*}{ Ref. [4] } & 3 & isotropic & $9.0 \times 10^{-5}$ \\
\cline { 2 - 4 } & 1 & beam like & $3.8 \times 10^{-6}$ \\
\hline \multirow{2}{*}{ Ref. [5] } & - & isotropic & 0.75 \\
\cline { 2 - 4 } & - & isotropic & 7.5 \\
\hline
\end{tabular}

\section{Search Time Window for Solar Flare Neutrinos}

Since neutrinos are generated via the decay of charged pions, the energy spectra have peak around $50-70 \mathrm{MeV}$. Therefore, the most interested energy region for solar flare neutrino event is from $10-500 \mathrm{MeV}$, which typically overlapped with the energy range of atmospheric neutrinos [6] and Supernova Relic Neutrinos (SRNs) [7]. The separation between solar flare neutrinos and atmospheric neutrinos is technically difficult because of the same production mechanism. Although atmospheric neutrinos are constantly generated, solar flare neutrinos are expected to be released 
only during the time scale of particle acceleration in solar flare. Thus, the ratio of signal (solar flare neutrinos) to background (atmospheric neutrinos) can be improved by setting an appropriate search time window. For this purpose, we firstly set the search time windows for searching for solar flare neutrinos by using the data recorded by the satellites. After the determination of the time windows, solar flare neutrinos were sought using the SK data taken from April 1996 to May 2018.

\subsection{Solar Flares on the Visible Side}

To search for solar flare neutrinos from visible side of the Sun, the public data from solar satellites GOES [8], RHESSI [9] and GEOTAIL [10], and computational resources of the Center for Integrated Data Science (CIDAS) at Nagoya University were used. In this study, the solar flares with more than X5.0 class were selected and the number of solar flares used for the analysis is 23. The solar flares are summarized in Table 2. The time of the start and end of each flare were determined using the time profile of soft X-ray derivative using GOES, see Ref. [11] for more detailed method.

Table 2: Summary of solar flares used for the search. The date, class and active region (AR) location are taken from a National Oceanic and Atmospheric Administration source (NOAA [12]). All time information is written in Universal Time Coordinate (UTC).

\begin{tabular}{|c|c|c|c|c|c|c|c|}
\hline Date & Class & AR location & $\begin{array}{c}\text { Start } \\
\text { (UTC) }\end{array}$ & $\begin{array}{l}\text { End } \\
\text { (UTC) }\end{array}$ & $\begin{array}{c}\text { Visible energy of } \\
\text { observed signal }\end{array}$ & $\begin{array}{l}\text { Event time } \\
\text { (UTC) }\end{array}$ & $\begin{array}{c}\text { Fluence limit } \\
\Phi_{90}^{\mathrm{HIGHE}}\left[\mathrm{cm}^{-2}\right]\end{array}$ \\
\hline 1997 Nov. 6 & $\mathrm{X} 9.5$ & S18W63 & $11: 52: 13$ & $11: 54: 58$ & No signal & - & $5.6 \times 10^{5}$ \\
\hline 2000 Jul. 14 & X5.7 & N22W07 & 10:08:44 & $10: 28: 31$ & No signal & - & $5.6 \times 10^{5}$ \\
\hline 2001 Apr. 2 & $\mathrm{X} 20.0$ & S21E83 & $21: 35: 10$ & $21: 52: 57$ & No signal & - & $5.6 \times 10^{5}$ \\
\hline 2001 Apr. 6 & X5.6 & S21E31 & $19: 12: 32$ & 19:21:31 & No signal & - & $5.6 \times 10^{5}$ \\
\hline 2001 Apr. 15 & $\mathrm{X} 14.4$ & S20W85 & $13: 43: 48$ & 13:51:03 & No signal & - & $5.6 \times 10^{5}$ \\
\hline 2001 Aug. 25 & X5.8 & S17E34 & $16: 28: 36$ & $16: 33: 38$ & No signal & - & $5.6 \times 10^{5}$ \\
\hline 2001 Dec. 13 & $\mathrm{X} 5.3$ & N16E09 & $14: 24: 25$ & 14:31:05 & No signal & - & $5.6 \times 10^{5}$ \\
\hline 2002 Jul. 23 & $\mathrm{X} 5.1$ & S13E72 & $00: 25: 08$ & 00:31:36 & No SK data ${ }^{1}$ & - & - \\
\hline 2003 Oct. 23 & X5.4 & S21E88 & $08: 20: 32$ & 08:36:03 & No signal & - & $5.6 \times 10^{5}$ \\
\hline 2003 Oct. 28 & $\mathrm{X} 17.2$ & S16E08 & $10: 59: 30$ & 11:10:57 & No signal & - & $5.6 \times 10^{5}$ \\
\hline 2003 Oct. 29 & $\mathrm{X} 10.0$ & S15W02 & $20: 38: 46$ & 20:50:07 & No signal & - & $5.6 \times 10^{5}$ \\
\hline 2003 Nov. 2 & X9.2 & S14W56 & $17: 12: 01$ & $17: 26: 03$ & No signal & - & $5.6 \times 10^{5}$ \\
\hline 2003 Nov. 4 & $\mathrm{X} 28.0$ & S19W83 & $19: 36: 59$ & 19:56:03 & $181.9 \mathrm{MeV}$ & $19: 42: 26$ & $8.5 \times 10^{5}$ \\
\hline 2005 Jan. 20 & X7.1 & N14W61 & $06: 39: 22$ & $06: 57: 12$ & No signal & - & $5.6 \times 10^{5}$ \\
\hline 2005 Sep. 7 & $\mathrm{X} 18.2$ & S11E77 & $17: 23: 34$ & $17: 39: 48$ & No signal & - & $5.6 \times 10^{5}$ \\
\hline 2005 Sep. 8 & X5.4 & S12E75 & $21: 00: 27$ & 21:08:05 & No signal & - & $5.6 \times 10^{5}$ \\
\hline 2005 Sep. $9^{2}$ & X6.2 & S12E67 & 19:13:00 & 21:20:36 & No signal & - & $5.6 \times 10^{5}$ \\
\hline 2006 Dec. 5 & $\mathrm{X} 9.0$ & S07E68 & $10: 24: 18$ & $10: 35: 43$ & No signal & - & $5.6 \times 10^{5}$ \\
\hline 2006 Dec. 6 & X6.5 & S05E64 & $18: 41: 29$ & 18:47:06 & No signal & - & $5.6 \times 10^{5}$ \\
\hline 2011 Aug. 9 & X7.4 & N14W69 & 08:00:50 & 08:05:40 & No signal & - & $5.6 \times 10^{5}$ \\
\hline 2012 Mar. 7 & X5.4 & N22E12 & $00: 04: 21$ & $00: 25: 16$ & No signal & - & $5.6 \times 10^{5}$ \\
\hline 2017 Sep. 6 & X9.4 & S09W34 & $11: 54: 39$ & 12:03:20 & $1186.8 \mathrm{MeV}$ & 12:03:05 & $8.9 \times 10^{5}$ \\
\hline 2017 Sep. 10 & X8.3 & S08W88 & $15: 49: 12$ & 16:06:32 & No signal & - & $5.6 \times 10^{5}$ \\
\hline
\end{tabular}

${ }^{1}$ It was a period of reconstruction work of SK.

2 It was failed to obtain the time window determined by derivative of soft X-ray for this solar flare. For this flare, the search time window is set by using soft X-ray time profile. Further discussion can be found in Ref. [11].

\subsection{The Invisible Side Solar Flares}

As described in Sec. 1, the predicted neutrino flux originated from the solar flares occurred on the invisible side would be larger than that of visible side. To obtain the information of solar flares 
on the invisible side, Coronal Mass Ejection (CME) data was used. Although the states on the solar surface of the invisible side is not observed directly, the start time of solar flares can be estimated with CME data recorded by LASCO [14]. There is weak positive correlation between solar flare intensity and CME speed.

At first, we selected 10 CMEs occurred on the invisible side of the Sun, whose speed exceeds $2000 \mathrm{~km} / \mathrm{sec}$. This criterion roughly corresponds to the solar flare with class X2.0 according to statistical evaluation [15]. In addition, the difference of the start time between CMEs and solar flares is also examined and the standard deviation of its distribution is 1020 seconds $(1 \sigma)$. Based on this study, we set the start time of the search window as 3060 seconds $(3 \sigma)$ before the CMEs emission. For the end time, we conservatively used 4178 seconds determined from X-ray described in Subsection 2.1 since all processes (acceleration, energy release and etc) occurs during a solar flare complete within this time duration. The solar flares occurred on the invisible side are summarized in Table 3.

Table 3: Summary of solar flares occurred on the invisible side. The date, CME speed and location are taken from SOHO LASCO CME catalog [16]. All time information is written in Universal Time Coordinate (UTC).

\begin{tabular}{|c||c|c|c|c|c|c|c|}
\hline Date & $\begin{array}{c}\text { CME speed } \\
{[\mathrm{km} / \mathrm{s}]}\end{array}$ & AR location & $\begin{array}{c}\text { Start } \\
\text { (UTC) }\end{array}$ & $\begin{array}{c}\text { End } \\
\text { (UTC) }\end{array}$ & $\begin{array}{c}\text { Visible energy of } \\
\text { observed signal }\end{array}$ & $\begin{array}{c}\text { Event time } \\
\text { (UTC) }\end{array}$ & $\begin{array}{c}\text { Fluence limit } \\
\Phi_{90}^{\text {HIGHE }}\left[\mathrm{cm}^{-2}\right]\end{array}$ \\
\hline 2001 Apr. 18 & 2464.2 & SW90b & $01: 15: 24$ & $03: 16: 02$ & No signal & - & $5.6 \times 10^{5}$ \\
\hline 2002 Jul. 19 & 2191.3 & E90b & $18: 07: 20$ & $20: 07: 58$ & No SK data $^{1}$ & - & - \\
\hline 2002 Jul. 20 & 2046.6 & S15E90 & $15: 13: 34$ & $17: 14: 12$ & No SK data $^{1}$ & - & - \\
\hline 2003 Nov. 2 & 2036.0 & SW90b & $08: 09: 23$ & $10: 10: 01$ & No signal & - & $5.6 \times 10^{5}$ \\
\hline 2003 Nov. 7 & 2237.0 & W90b & $14: 41: 19$ & $16: 41: 57$ & $\begin{array}{c}3555.9 \mathrm{MeV} \\
493.1 \mathrm{MeV}\end{array}$ & $\begin{array}{c}15: 18: 34 \\
15: 40: 45\end{array}$ & $1.2 \times 10^{6}$ \\
\hline 2003 Nov. 9 & 2008.1 & E90b & $05: 06: 57$ & $07: 07: 35$ & No signal & - & $5.6 \times 10^{5}$ \\
\hline 2005 Jul. 24 & 2527.8 & E90b & $12: 44: 51$ & $14: 45: 29$ & $125.5 \mathrm{MeV}$ & $13: 07: 36$ & $1.2 \times 10^{6}$ \\
& & & & & $1351.3 \mathrm{MeV}$ & $14: 15: 21$ & \\
\hline 2011 Jun. 4 & 2425.5 & N16W153 & $20: 51: 42$ & $22: 52: 20$ & $2135.6 \mathrm{MeV}$ & $22: 09: 37$ & $8.2 \times 10^{5}$ \\
\hline 2012 Jul. 23 & 2003.2 & S17W132 & $01: 19: 07$ & $03: 19: 45$ & $834.8 \mathrm{MeV}$ & $03: 03: 47$ & $8.2 \times 10^{5}$ \\
\hline 2014 Dec. 13 & 2221.6 & S20W143 & $13: 06: 34$ & $15: 07: 12$ & No signal & - & $5.6 \times 10^{5}$ \\
\hline
\end{tabular}

${ }^{1}$ It was a period of reconstruction work of SK.

\section{Analysis of Super-Kamiokande Data}

SK is a water Cherenkov detector located at $1,000 \mathrm{~m}$ (2,700 $\mathrm{m}$ water equivalent) below the top of Mt. Ikenoyama in Gifu prefecture, Japan [17]. Search for solar flare neutrinos was performed using the analysis tools in SK. The energy region of this analysis are divided into two energy regions, above and below $100 \mathrm{MeV}$ (High-energy data sample and low-energy data sample). The livetime and background rate of each sample are summarized in Table 4.

\subsection{High-energy Data Sample}

The high-energy data sample has three different categories: fully contained (FC) neutrinos, partially contained (PC) neutrinos, and upward-going muon (UPMU). See Ref. [18] for more detailed information of the event categories. In this study, the FC data sample was used since the peak of expected energy spectrum of solar flare neutrinos is around $70 \mathrm{MeV}$, and the energy range of the FC sample is $100 \mathrm{MeV}$ to $10 \mathrm{GeV}$. 


\subsection{Low-energy Data Sample}

Low-energy data sample can be categorized into two data sets, solar neutrino data [19] and SRNs data [7]. The SRNs data were used for the solar flare neutrino analysis because the energy range of the SRNs data is from 16 to $100 \mathrm{MeV}^{1}$. Further information about the reconstruction methods and event selection can be found in Kirk et al. [7].

Table 4: Summary for SK data set

\begin{tabular}{|c||c|c|c|c|c|}
\hline \multicolumn{1}{|c||}{ SK Phase } & Data period & \multicolumn{2}{c|}{ High energy data sample } & \multicolumn{2}{c|}{ Low energy data sample } \\
\hline & & livetime & background event rate & livetime & background event rate \\
\hline SK-I & Apr. 1996 - Jun. 2001 & 1489 days & $7.45 \pm 0.07$ event/day & 1497 days & $0.20 \pm 0.01$ event/day \\
\hline SK-II & Oct. 2002 - Oct. 2005 & 825 days & $7.33 \pm 0.09$ event/day & 794 days & $0.19 \pm 0.02$ event/day \\
\hline SK-III & Jul. 2006 - Sep. 2008 & 522 days & $7.53 \pm 0.12$ event/day & 562 days & $0.20 \pm 0.01$ event/day \\
\hline SK-IV & Sep. 2008 - May 2018 & 3235 days & $7.48 \pm 0.05$ event/day & 2970 days & $0.19 \pm 0.01$ event/day \\
\hline
\end{tabular}

\section{Result}

\subsection{Visible Side Solar Flares}

There is no signal detected in low energy data sample while two events in the high energy data sample detected as summarized in Table 2. Figure 1 shows the time of observed events together with light curves recorded by GOES. Figure 2 (left) shows the energy of observed event with the background energy spectrum. The expected number of background events of high-energy data samples in the search time windows is 0.58 events ( 0.29 events) for solar flare event on November 4th 2003 (September 6th 2017). The probability of no signal event is $32.2 \%$ (21.8\%) which is consistent with background.

\subsection{Invisible Side Solar Flares}

There is no signal in low-energy data sample while six events in high-energy data sample as summarized in Table 3. Figure 3 shows the energy and time of observed events with search time window described in Sec 2. Figure 2 (right) shows the energy of observed event with the background energy spectrum. The expected number of background events of high-energy data sample is 0.62 event/flare. It is the same for all the solar flares on the invisible side since the search time range is uniform for the solar flares on the invisible side. The numbers of observed events in search time window are two events for the solar flares on November 7th 2003 and July 24th 2005. The probabilities of no signal event for these solar flares are $10.2 \%$. These are the most extreme cases, but they are consistent with background.

\subsection{Fluence limit using Fagion's and Moscat's Model}

The number of neutrino candidate events observed in the search window can be converted to the upper limit on the neutrino fluence. In this study, the limits of the solar flare neutrinos were calculated using the Fagion's and Moscat's prediction. The 90\% C.L. limits on the fluence of solar

\footnotetext{
${ }^{1}$ The solar angle cut was used for the SRNs searches, however, this cut was not used for the solar flare neutrino analysis.
} 

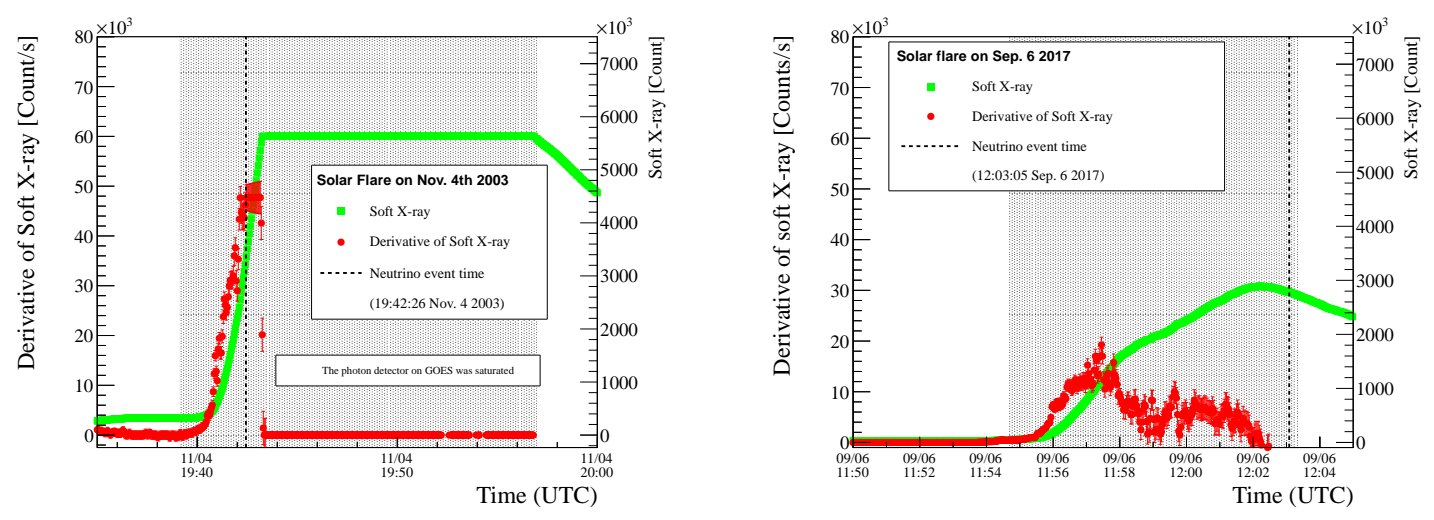

Figure 1: Neutrino event timing on soft $X$-ray light curve and its time derivative recorded by GOES. Green square (Red point) shows count rate of soft X-ray (time derivative of soft X-ray). Black dashed line shows neutrino event timing. Gray band is corresponding to search time window. The flat count rate on left plot is due to saturation of photon detector on GOES from 19:43:18 to 19:56:51 on November 4th 2003.
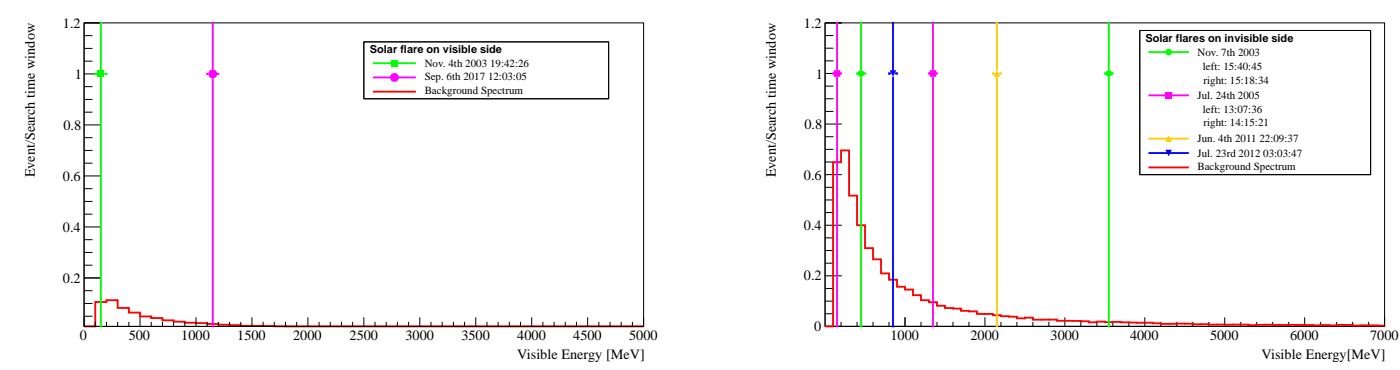

Figure 2: Left (right) plot is energy spectra of background and observed events in search time window of solar flare on visible (invisible) side. Red histogram shows background energy spectrum. Green, magenta, blue and orange lines show the energies of the neutrino events in search time windows from each solar flare, respectively.

neutrinos can be calculated using the following equation

$$
\Phi_{90}^{\mathrm{LOWE}}=\frac{N_{90}}{N_{p} \int \mathrm{d} E_{\nu} F\left(E_{\nu}\right) \sigma\left(E_{\nu}, E_{e^{+}}\right) \epsilon\left(E_{\nu}\right)}
$$

where $N_{90}$ is the $90 \%$ C.L. limit of the observed neutrino events calculated with a Poisson distribution with the number of background events, $N_{p}$ is the number of target proton in SK relevant to the neutrino interactions, $\sigma$ is the Invers Beta Decay (IBD) cross section which is used in event simulaition. Since there was no signal in low energy data samples, $N_{90}$ is 2.30 for all flare events. Assuming the energy spectrum of solar flare neutrino is Fargion's and Moscat's prediction [5], the fluence limit for the low energy sample is $1.7 \times 10^{7} \mathrm{~cm}^{-2} /$ flare.

For the high energy data set, the fluence limit of solar flare neutrino can be obtained using the equation

$$
\Phi_{90}^{\mathrm{HIGHE}}=\frac{N_{90}}{N_{T} \int \mathrm{d} E_{\nu} \sum_{i=e, \mu, \tau, \bar{e}, \bar{\mu}, \bar{\tau}} 1 / 6\left(F\left(E_{v_{i}}\right) \sigma\left(E_{v_{i}}\right) \epsilon\left(E_{v_{i}}\right)\right)}
$$



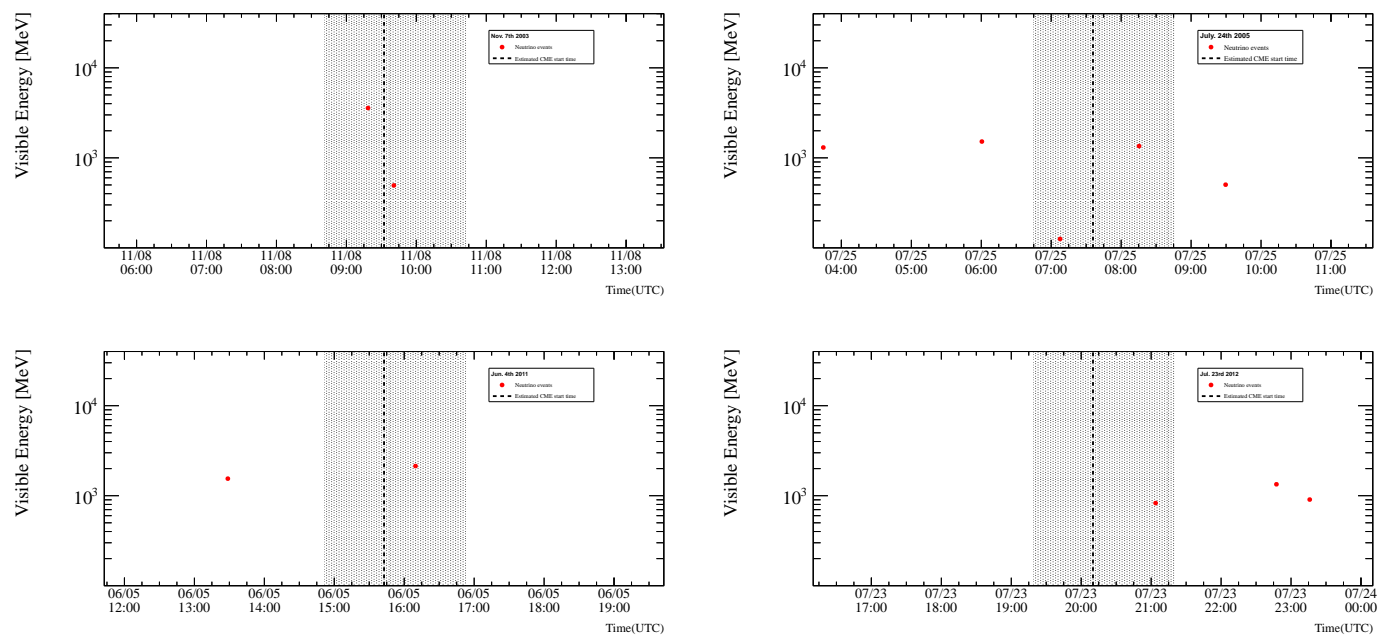

Figure 3: Time distribution of neutrino events in high energy data sample for solar flares on invisible side. Red points show neutrino event in SK. Grey band shows the search time window. Black dashed line is corresponding to estimated start time of CME emission.

where $N_{T}$ is the number of target nuclei relevant to the neutrino interactions, $\sigma$ is the combined cross section for all interactions. The $90 \%$ C.L. upper limits of the fluence for solar flare neutrino in the high energy data sample in each flare are summarized in Table 2 (visible side) and Table 3 (invisible side). Assuming that the search time windows are sufficient to cover the neutrino emission time in solar flares, the fluence limit does not depend on the duration of search time window.

\subsection{Model independent fluence upper limit}

To compare the results of this work with the other experimental results, the model independent fluence upper limit was also calculated for the low energy sample using the equation

$$
\Phi_{90}(E)=\frac{N_{90}}{N_{p} \int \mathrm{d} E_{v} \delta\left(E-E_{v}\right) \sigma\left(E_{\nu}, E_{e^{+}}\right) \epsilon\left(E_{v}\right)}
$$

where $\delta\left(E-E_{v}\right)$ is delta function and other variables are same as that in Eq. (1). The same procedure laid out in Ref. [20] was used to calculate the values. Figure 4 shows the results of the upper limits of fluence for solar flare neutrinos with model independent method. Comparing to the Kamiokande-II result, the sensitivity was improved by an order of magnitude in the energy region from 20 to $110 \mathrm{MeV}$.

\section{Summary and discussion}

For solar flares on the visible and invisible sides, we estimated the time of neutrino emission from optical and CME observations, respectively. Then, we searched for neutrino events associated with the solar flares in Super-Kamiokande. Two neutrino events from solar flares on the visible side and six neutrino events from solar flares on the invisible side are observed, but all of them are background consistent, so the upper limit of the solar flare neutrino fluence is calculated. The 


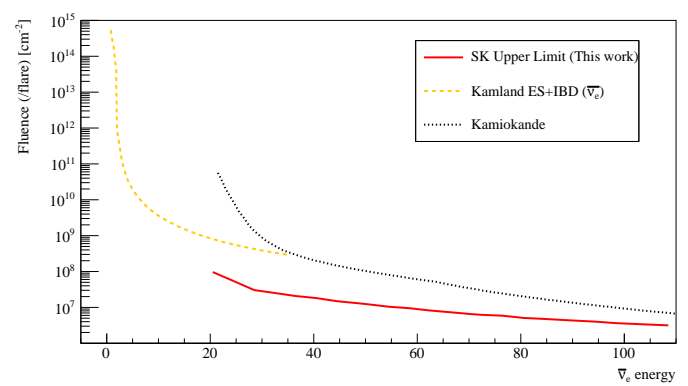

Figure 4: Model independent upper limit for solar flare neutrino search. Red solid line shows the upper limit calculated from this result. Black dotted (Orange dashed) line shows the Kamiokande (KamLAND) result [2].

obtained result is the strong constraint of the fluence of solar flare neutrinos in $\mathrm{MeV}$ region up to date. More detailed analysis is on-going and the results are presented in the future publication.

\section{References}

[1] Hudson, H., Ryan, J.: 1995, Annu. Rev. Astron. Astrophys. 33, 239.

[2] Davis, R.: 1994, Prog. Part. Nucl. Phys. 32, 13.; Hirata, K.S., et al.: 1988, Phys. Rev. Lett. 61, 2653. ; Aglietta, M., et al.: 1991, APJ 382, 344. ; Aharmim, B., et al.: 2014, Astropart. Phys. 55, 1.; Agostini, M., et al.: 2019, arXiv:1909.02422.; Abe, S., et al.: 2021, arXiv:2105.02458. ;Abbasi, R, et al.: 2021, Phys. Rev. Lett. 103, 102001.

[3] Kocharov, G.E., Kovaltsov, G.A., Usoskin, I.G.: 1991, Nuvo Cim. C 14, 417.

[4] Takeishi, R., et al.: 2013, Proceedings of 33rd ICRC 2013, 3656.

[5] Fargion, D., Moscato, F.: 2003, Chin. J. Astron. Astrophys. 3, 75.

[6] Abe, K., et al.: 2018, Phys. Rev. Lett. 97, 072001.

[7] Kirk, B., et al.: 2012, Phys. Rev. Lett. 85, 052007.

[8] Hanser, F.A., Sellers, F.B.: 1996, Proc. SPIE. 2812, 344.

[9] Lin, R.P., Dennis, B.R., Hurford, D.M.: 2002, Solar Phys. $210,3$.

[10] Tanaka, Y.T., et al.: 2007b, Rev. Sci. Instrum. 78, 034501.

[11] Okamoto, K., et al.: 2020, Solar Phys. 295, 10.

[12] Solar flare catalog based on data recorded by GOES, available at https://www.ngdc.noaa.gov/stp/space-weather/solar-data/solar-features/ solar-flares/x-rays/goes/xrs/, date last accessed July 12021.

[13] Davies, J.A., et al. : 2009, Geophysical Research Letters 36, 2.

[14] Brueckner, G. E., et al.: 1995, Solar Phys. 162, 1-2, pp. 357-402.

[15] Yashiro, S., et al.: 2008, Proceedings of the International Astronomical Union 4(S257), 233-243.

[16] CME catalog based on data recorded by LASCO, available at https://cdaw.gsfc.nasa.gov/CME_list/, date last accessed July 12021.

[17] Fukuda, S., et al.: 2003, Nucl. Instrum. Meth. A 501, 418.

[18] Ashie, Y., et al.: 2005, Phys. Rev. Lett. 71, 112005.

[19] Abe, K., et al.: 2016, Phys. Rev. Lett. 94, 052010.

[20] Abe, K., et al.: 2018, Astrophys. J. Lett. 857, L4. 